\title{
Repercusiones oculares ante la sospecha de trauma craneal
} abusivo

\section{Ocular repercussions of suspected abusive head trauma}

Luis P. Orozco-Gómez ${ }^{a *}$, Julio Martínez-Almada ${ }^{b}$, Luis G. Moreno-Ferreyrac, Leonor Hernández-Salazaro, Andrés Orozco-Mogueld ${ }^{d}$ Alberto Dávila-Bárcena ${ }^{e}$ y Juan C. Medina-Díaz ${ }^{f}$

a Servicio de Oftalmología, Centro Médico Nacional 20 de Noviembre, Instituto de Seguridad y Servicios Sociales de los Trabajadores del Estado (ISSSTE), Ciudad de México; ${ }^{b}$ Departamento de Vitreo y Retina, Centro Médico Nacional 20 de Noviembre, ISSSTE, Ciudad de México; 9 Unidad Médica de Alta Especialidad T1, Instituto Mexicano del Seguro Social (IMSS), León, Guanajuato; 'Hospital General de La Paz, IMSS, Baja Califörnia; ${ }^{e}$ Departamento de Retina, Hospital Regional General Ignacio Zaragoza, ISSTE, Ciudad de México; ${ }^{f}$ Departamento de Retina, Hospital Mexiquense de la Salud Visual del Estado de México, Naucalpan de Juarez, Estado de México. México

\section{Resumen}

Objetivo: Mostrar los hallazgos oculares de una serie de casos con sospecha de trauma craneal abusivo, su tratamien̆to y las posibles secuelas. Método: Estudio retrospectivo, serie de casos de pacientes menores de edad con sospechâ de trauma craneal abusivo que presentaron crisis convulsivas por encefalopatía, hemorragias intrarretinianas y otras alterăciones sistémicas sugestivas de síndrome del niño sacudido. Resultados: Se incluyeron 8 pacientes, de los cuales 3 (38\%) eran del sexo femenino y 5 (62\%) del sexo masculino. En todos ellos, el servicio de oftalmología acudió como intercon̄sultante. El motivo de consulta fue por crisis convulsivas en 7 (87\%) casos. La edad media de presentación fue de 4.12 messes ( \pm 2 meses). La persona que presenció los síntomas del infante fue la madre en el $57 \%$ de los casos. Cuatro de los pacientes $(50 \%)$ presentaron fracturas (costales, craneales, tibiales, acromiales y claviculares), todas ellas diagnosticadas por estudios radiológicos. El 100\% de los pacientes sufrieron lesiones intraoculares bilaterales: hemorragias intrarretinianas (100\%), hemorragia vítrea (36\%) y agujero macular postraumático (21\%). El 29\% requirió vitrectomía. Conclusiones: La observąción de hemorragia subdural, hemorragia retiniana y encefalopatía se considera indicador de sacudida o maltrato de bèbés, habitualmente menores de 1 año. El hallazgo de la tríada no hace el diagnóstico, pero es altamente sugestivo de este. en gran parte por la ausencia de otras explicaciones congruentes con los hallazgos clínicos. La base científica para la afirmación de que las lesiones son consecuencia de una violenta sacudida intencionada es altamente discutible y las repercúsiones legales pueden tener consecuencias insospechadas.

Palabras clave: Daño encefálico. Hemorragia cerebral. Maltrato al niño. Retinopatía. Traumatismo craneocerebral.

\section{Abstract}

Purpose: To determine the ophthalmological findings in a case series of suspected abusive head trauma, as well as its sequels and treatment. Methods: We conducted a retrospective case series of patients under one year of age with suspected history of abusive head trauma caused by shaking, direct trauma, or with a clinical presentation of seizures due to-encephalopathy, retinal hemorrhage, or any other systemic repercussions suggestive of the shaken baby syndrome. Results:

\section{Correspondencia:}

*Luis P. Orozco-Gómez

San Francisco, 1626-1605

Col. del Valle, Del. Alcaldía Benito Juárez

Fecha de recepción: 24-09-2019

C.P. 03100 , Ciudad de México, México

E-mail: Ip.orozco@issste.gob.mx

Fecha de aceptación: 22-08-2020

DOI: 10.24875/RMO.M20000145
Disponible en internet: 11-01-2021 Rev Mex Oftalmol. 2021;95(1):20-27 www.rmo.com.mx 0187-4519/@ 2020 Sociedad Mexicana de Oftalmología. Publicado por Permanyer. Este es un artículo open access bajo la licencia CC BY-NC-ND (http://creativecommons.org/licenses/by-nc-nd/4.0/). 
The study included 8 subjects, of which 5 were male (62\%) and 3 female (38\%), with a mean age of 4.12 months ( \pm 2 months). All of them were examined by the ophthalmology department. In 7 cases (87\%), the reason for consultation was seizures. In $57 \%$ of the studied cases, the mother was the person who witnessed the symptoms. Rib, skull, tibia, acromion, and collarbone fractures were radiologically diagnosed on $4(50 \%)$ patients. All patients (100\%) had bilateral vitreoretinal involvement including intraretinal haemorrhage (100\%), vitreous hemorrhage (36\%) and post-traumatic macular hole (27\%). Vitrectomy was performed in $29 \%$. Conclusions: The combination of subdural hemorrhage, retinal hemorrhage and encephalopathy in subjects under one year of age, is considered indicative of abusive, inflicted or non-accidental head injury. While the triad can not confirm the diagnosis of the shaken baby syndrome, these findings are highly suggestive especially due to the lack of other explanations consistent with the clinical findings. It is highly disputable whether this diagnostic triad can be used as a confirmatory tool for an abusive direct trauma or both, and the legal consequences may be unsuspected.

Key words: Brain damage. Cerebral hemorrhage. Child abuse. Retinopathy. Cranial trauma.

\section{Introducción}

El síndrome del niño sacudido tradicionalmente ha sido definido como un conjunto de lesiones resultantes de un movimiento de flexión-extensión de la cabeza y el cuello súbito y repetitivo, provocado en un niño generalmente menor de 2 años, quien es sujetado del tronco por otra persona y esta lo sacude violentamente. Este movimiento genera una aceleración y desaceleración de la cabeza que favorece el desarrollo de hemorragias intrarretinianas, hemorragias intracraneanas, fracturas ocultas, hematomas sobre la piel e incluso lesión en el centro de la respiración a nivel del bulbo raquídeo, provocando la muerte de la víctima por paro respiratorio ${ }^{1,2}$. Las secuelas graves en los sobrevivientes no son poco frecuentes.

Esta patología es poco reconocida por el personal médico responsable de su atención, por lo que su incidencia es poco conocida, pero se estima que se presentan unos 1,200-1,600 casos al año, con una mortalidad de hasta el $25 \%{ }^{3}$.

Recientemente la nomenclatura intenta ser más precisa y se está modificando a trauma craneal abusivo, lesión cerebral adquirida o lesión por sacudida e impacto, ya que la base científica para la afirmación de que esas lesiones son consecuencia de una violenta sacudida intencionada es altamente discutible ${ }^{4}$. La evidencia biomecánica ha mostrado que la sacudida sin contacto solo produciría la tríada de lesiones (hemorragia subdural, hemorragia retiniana y encefalopatía), junto con otras lesiones en el cuello y la médula espinal que típicamente no se encuentran en supuestos casos de niños sacudidos. Las lesiones intraoculares suelen ser vitreorretinianas, entre las que se encuentran agujeros maculares, hemorragias vítreas, retinosquisis, desprendimientos de retina (que pueden ser traccionales o mixtos) y hemorragias intrarretinianas, que suelen ser las lesiones más comunes 5,6 .
La mayor parte de la literatura al respecto está enfocada en el área pediátrica más que en la oftalmológica; internacionalmente se reporta una incideñcia del $17-24 \%$. 7 . En México no se puede precisar sưfrecuencia porque habitualmente no se establece el diagnóstico en los hospitales adonde son llevados estos niños para su atención, y por ello no se registra.

Se piensa que el mecanismo por el cual se genēran las lesiones es una violenta sacudida de la cabeza, que al estar sostenida por la débil musculatura del cuello permite que vaya hacia delante y atrás sin control. Estos movimientos causan hipoxia por afectación del centro respiratorio en el bulbo raquídeo, edema y hemorragia cerebral, así como hemorragia retinianā de localización y gravedad variables ${ }^{9,10}$. En algunos càsos también ocurre desprendimiento del vítreo posteriö, lo que ocasiona tracción de su adherencia maculâr y provoca un agujero macular y posteriormente desprendimiento de la retina.

En una tercera parte de los casos, el paciente también puede ser azotado contra una superficie dura provocándole fractura de la bóveda craneal y de otros huesos del cuerpo (síndrome del niño sacudido y con traumatismo) $)^{11}$.

Se ha descrito que el factor desencadenante de la agresión puede ser el llanto intenso e inconsolable, la irritabilidad constante o un rechazo persistente del hiño al alimento ${ }^{12,13}$. Es muy difícil precisar quién füẹ el agresor. La información se ha obtenido durante el je⿶凵icio legal seguido contra algunas personas sospechosas. El agresor es predominantemente del sexo masculino y puede ser el cuidador que, al inicio del cuadro de extrema gravedad, estaba con el menor ${ }^{14}$.

Las manifestaciones que habitualmente obligan ălos padres o a los cuidadores a solicitar atención médica de urgencia son la presentación súbita e inesperadá de crisis convulsivas, deterioro neurológico agudo o suffimiento cardiorrespiratorio crítico $^{15}$. Cuando existe la 
sospecha de síndrome del niño sacudido se requiere que el grupo médico de los servicios de medicina crítica (urgencias y terapia intensiva pediátrica), y los servicios interconsultantes (el servicio de oftalmología, entre otros), la consideren cuando un niño, habitualmente menor de 2 años, presenta dicho cuadro clínico.

A continuación hacemos una revisión de los casos diagnosticados y atendidos en el Centro Médico Nacional 20 de Noviembre, del Instituto de Seguridad y Servicios Sociales de los Trabajadores del Estado, que nos permitieron conocer y precisar el diagnóstico para su atención integral. El objetivo es mostrar una serie de casos valorados en un centro médico nacional cuya sospecha fue trauma craneal abusivo enfocado en los hallazgos oftalmológicos, así como el tratamiento ofrecido a estos pacientes y las secuelas del trauma craneal abusivo.

\section{Método}

Presentamos una serie de casos en la que se incluyeron todos los pacientes con diagnóstico presuntivo de síndrome de trauma craneal abusivo, entre enero de 2010 y diciembre de 2018. Para esta revisión se definió trauma craneal abusivo como la presencia de crisis convulsivas aunadas a hemorragia retiniana o cerebral, con o sin fracturas diversas, habiéndose descartado trastornos hematológicos. Se recabaron variables sociodemográficas y clínicas (antecedentes, cuidadores, cuadro clínico en el hogar, cuadro clínico y lesiones al ingreso al hospital, y evolución clínica). Se llevó a cabo el análisis descriptivo de las variables cuantitativas (promedio y mediana) y de las frecuencias relativas y absolutas para las variables cualitativas. No se realizaron las pruebas estadísticas que podrían permitir hacer inferencias de asociación entre las variables clínicas, pues la muestra es pequeña, por lo que únicamente se describen.

\section{Resultados}

Se incluyeron ocho pacientes, de los cuales $3(38 \%)$ eran del sexo femenino y 5 (62\%) del sexo masculino; el promedio de edad fue de 4.1 meses (Tabla 1). En el $100 \%$ de los casos, el servicio de oftalmología acudió como interconsultante al ser solicitado por el servicio de pediatría.

El motivo de consulta de los pacientes fue por crisis convulsivas en 6 (83.3\%). Otros síntomas que obligaron a acudir al hospital fueron dificultad respiratoria, rechazo al alimento, llanto inconsolable persistente, vómito, somnolencia y pérdida de la conciencia (Tabla 2 , Figs. 1 y 4).
Tabla 1. Edad y sexo de los pacientes

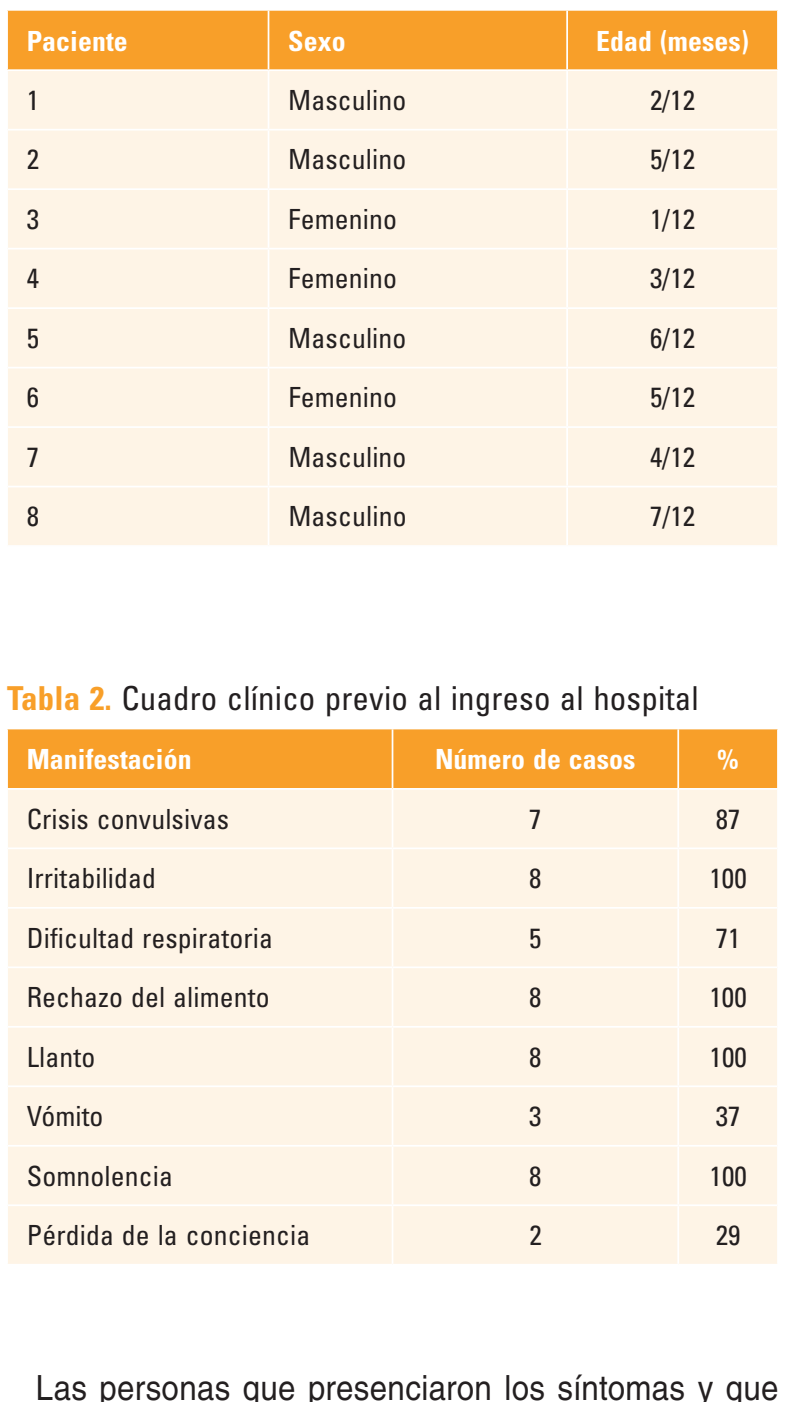
llevaron al niño a urgencias fueron muy variables, siendo la madre en el $57 \%$ de los casos (Tabla 3)

Entre los hallazgos de la exploración clínica encontramos que cuatro pacientes $(50 \%)$ presentaron fracturas en dištintas localizaciones: en el cráneo dos pacientes (uno ền la región parietal derechay otro en la región parietooccipital), un paciente presentó fracturas en cuatro costillas, ưno más presentó una fractura clavicular, y uno más tibiales y acromiales, todas ellas diagnosticadas por estüios radiológicos (Tabla 4, Figs. 2 y 3).

Entre los hallazgos oftalmológicos, todos los pacientes sufrieron lesiones intraoculares bilaterales: el $100 \%$ presentaron hemorragias intrarretinianas en ung 0 ambos ojos, el $36 \%$ presentaron hemorragia subhiäloidea, el $21 \%$ presentaron hemorragia vítrea de gradö IV y el $21 \%$ presentaron agujero macular postraumắtico (Tabla 5, Fig. 5). El 29\% de los pacientes requirió cirugía de vitrectomía vía pars plana por hemorragia vititrea persistente y agujero macular traumático (Fig. 6). 
Tabla 3. Personas que presenciaron los síntomas que llevaron al niño a urgencias

\begin{tabular}{|l|c|c|}
\hline Parentesco & Cuidador principal & $\%$ \\
\hline Solo madre & 4 & 57 \\
\hline Solo padre & 0 & 0 \\
\hline Ambos progenitores & 2 & 25 \\
\hline Tíos & 0 & 0 \\
\hline Abuelos & 1 & 14 \\
\hline Padrastro & 0 & 0 \\
\hline Cuidadora doméstica & 1 & 14 \\
\hline
\end{tabular}

Tabla 4. Otros hallazgos

\begin{tabular}{|l|c|c|}
\hline Datos clínicos & Nímero de pacientes & $\%$ \\
\hline Fractura tibial & 1 & 14 \\
\hline Fracturas costales & 1 & 14 \\
\hline Fracturas craneales & 2 & 29 \\
\hline Hematomas & 2 & 29 \\
\hline Mordida & 1 & 14 \\
\hline Impresión digital en la pierna & 1 & 7 \\
\hline Fractura acromial & 1 & 7 \\
\hline Fractura de clavícula & 1 & 7 \\
\hline Hemorragias intraparenquimatosas & 8 & 100
\end{tabular}

Tabla 5. Hallazgos oftalmológicos

\begin{tabular}{|l|c|c|}
\hline & Número de ojos & $\%$ \\
\hline Hemorragia intrarretiniana & 16 & 100 \\
\hline Hemorragia vítrea & 5 & 36 \\
\hline Agujero macular & 3 & 21 \\
\hline Desprendimiento de retina & 0 & 0 \\
\hline
\end{tabular}

\section{Discusión}

En la presente serie de casos, el $86 \%$ de los pacientes presentaron crisis convulsivas, lo que fue muy sugestivo de encefalopatía, el 100\% presentaron hemorragias intrarretinianas $y$, entre otros hallazgos, fracturas tibiales, costales, acromiales, craneales, hematomas y mordidas. Todos estos hallazgos en los pacientes nos hicieron sospechar un síndrome del niño maltratado.

Apoyándonos con Trabajo Social e interrogatorios dirigidos, los síntomas que precipitaron el trasladọ al hospital de estos niños fueron las crisis convulsivăs y las personas que presenciaron las crisis fueron las madres $(57 \%)$, ambos progenitores (14\%), la abøela (14\%) o una cuidadora (14\%).

Según la Organización Mundial de la Salud, el maltrato infantil se define como los abusos y desatenciones que reciben los menores de 18 años, incluido el maltrato físico, psicológico o sexual, que dañen su salud, desarrollo o dignidad, o bien que pongan en riesgô su supervivencia ${ }^{16}$.

Las consecuencias que vive un niño, niña o adolescẹnte que sufre maltrato empiezan desde que pasa por esa situación y pueden prolongarse por largo tiempo, incluso años o décadas, y llegar a comprometer su vida adulta.

En general, el niño maltratado no cumple con el desarrollo de las metas de su crecimiento y desarröllo, es decir, autoestima, felicidad, salud, creatividad, solidaridad, autonomía y espiritualidad, en un ambięnte bidireccional de construcción entre los padres y=los niños. Estas habilidades se ponen al servicio del fomento del tejido de la resiliencia, que es la capacidad humana para triunfar frente a la adversidad ${ }^{17}$.

Existen lesiones centinela que son definidas cồmo lesiones menores infringidas, las cuales son reconocidas por el médico o los padres, que son altamente sugèstivas de maltrato. Son comunes en niños maltratados físicamente y raras en los que no sufren maltrato. Se ha llegado a identificar su presencia previa en hasta un: $25 \%$ de los niños con maltrato físico y en un tercio de los diagnosticados de traumatismo craneoencefálico ${ }^{18}$.

Los indicadores típicos del abuso físico, en menör o mayor grado, son las magulladuras o moretones en điferentes fases de cicatrización y de forma extendidà en diferentes partes del cuerpo, las quemaduras con formas definidas, las fracturas de nariz o mandíbula, o en espiral de los huesos largos, las torceduras o dislocacionesplas heridas o raspaduras en la cara y la parte posteriớ de las extremidades y el torso, las señales de mordedüras humanas, los cortes o pinchazos, y las lesiones internas (en el cráneo o el cerebro, síntomas de asfixia.... $)^{19} \cdot \frac{\mathrm{C}}{\mathrm{O}}$

La presencia de hemorragia subdural, hemorraggia retiniana y encefalopatía se considera un indicador de sacudida del bebé, habitualmente en los menores de 1 año. El hallazgo de la tríada no hace el diagnóstico, pero es altamente sugestivo de este, en gran partepor la ausencia de otras explicaciones congruentes con̄los hallazgos clínicos ${ }^{20}$. 


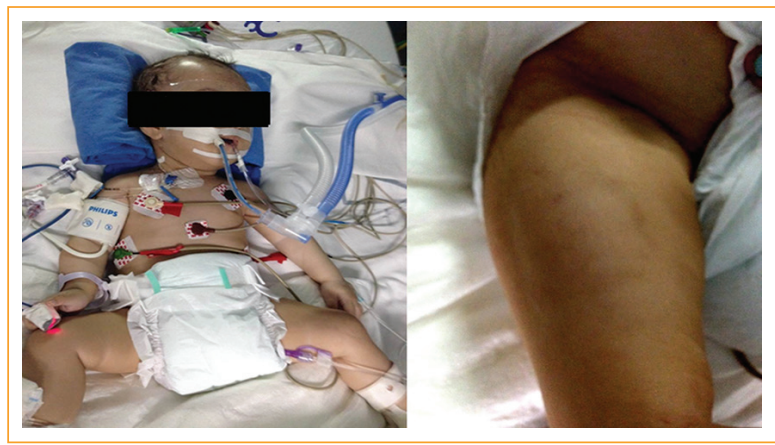

Figura 1. Paciente en coma barbitúrico con sospecha de trauma craneal abusivo, lesión con impresión de arcada dentaria en la pierna derecha.

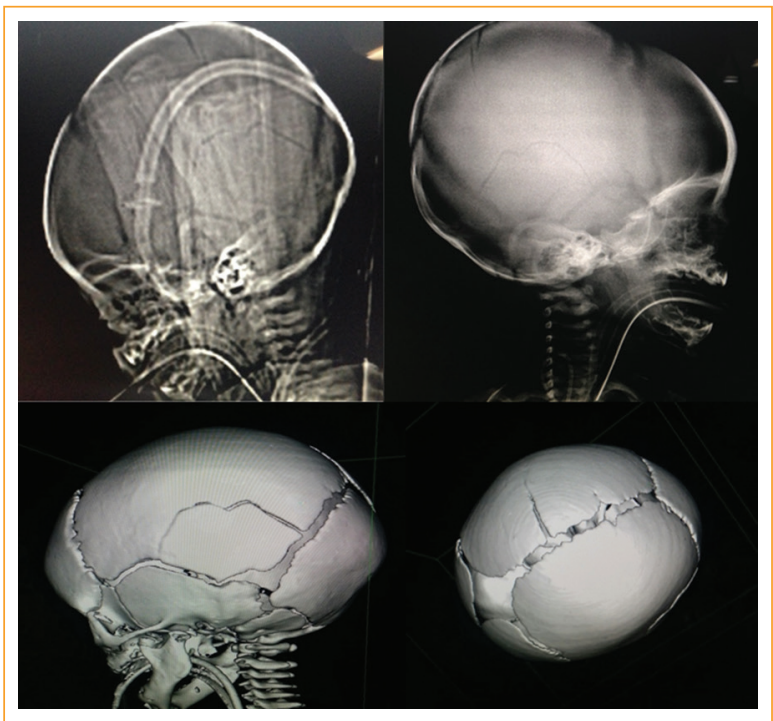

Figura 2. Radiografía simple y reconstrucción tridimensional del cráneo de uno de los pacientes con fractura craneal.

Si bien los médicos que pueden proponer el diagnóstico de síndrome del niño sacudido son los pediatras y los oftalmólogos por las interconsultas solicitadas basados en los hallazgos, esta es una opinión científica predominante, pero hay que recordar que dicho síndrome nunca ha sido probado como algo más que una hipótesis ${ }^{21}$.

Con frecuencia los especialistas ignoran las explicaciones alternativas que pueden proporcionar otras especialidades, o están en desacuerdo con ellas, o incluso con otros puntos de vista dentro de la misma especialidad. Más aún, existe un creciente número de artículos científicos revisados por otros expertos que desafían todo el concepto ${ }^{22}$.

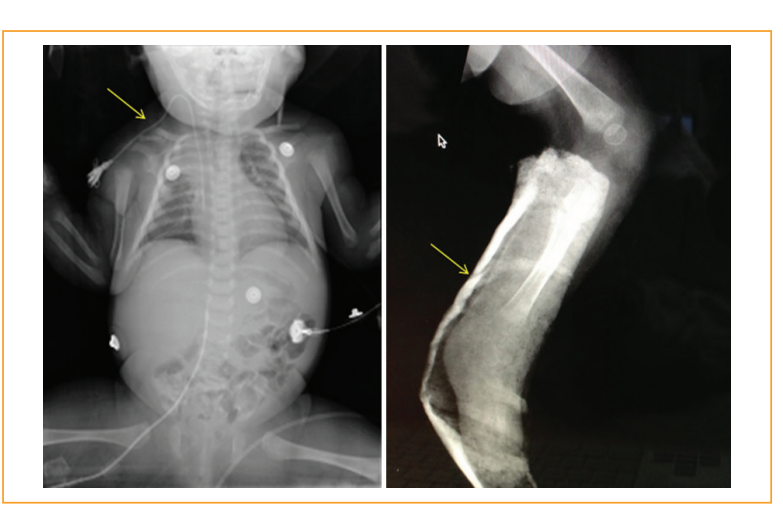

Figura 3. Radiografías que muestran fracturas clavicualar, acromial y tibial.
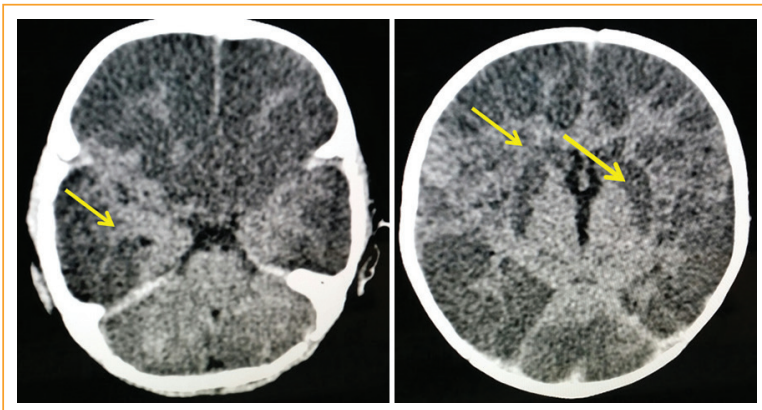

Figura 4. Edema cerebral con deterioro rostrocaudalo más evidente en el hemisferio derecho.

Notablemente, el requisito para una evidenciaccon base científica es mucho más riguroso en casos- de negligencia médica que en los juzgados de familitia o penales, donde creer que algo es verdad parece häber adquirido suficiente valor de evidencia para inclinablas determinaciones del tribunal ${ }^{23}$.

El síndrome del niño sacudido está teniendo cạda vez más una crítica significativa, ya que carece de convalidación científica y de rigor forense. Hasta la fecha, la investigación realizada genera una duda č̀nsiderable; más aún, mientras se sigan realizando dỉagnósticos usando este término, se priva a los bebés de las investigaciones necesarias para establecer la causa correcta, el tratamiento y la prevención dẹ su recurrencia, síntomas y señales, ya que la mayoría de los diagnósticos no se basan en un peritaje sino en la descripción de hallazgos hecha por pediatras y oftalmólogos ${ }^{4}$. Desde el punto de vista judicial, muchas consideraciones se referirán a una larga investigación, y se puede aplicar adecuadamente a los niños mẫtratados. El resultado de un juicio tendrá consecuencias 


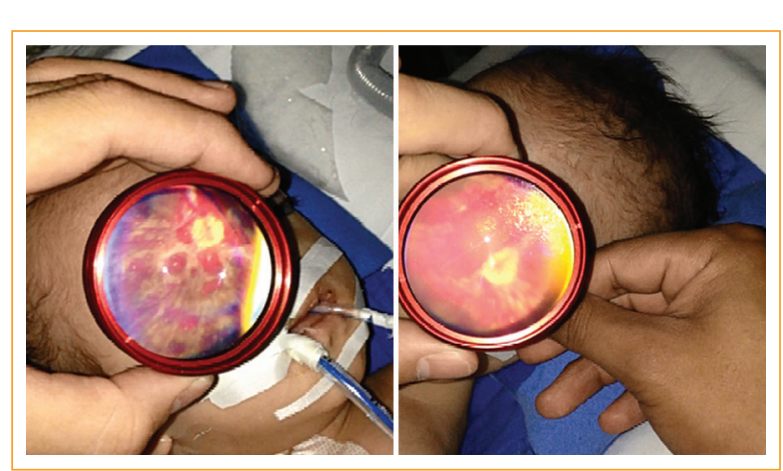

Figura 5. Imagen obtenida con un teléfono celular inteligente de fondo de ojo de ambos ojos de un paciente en la unidad de terapia intensiva pediátrica.

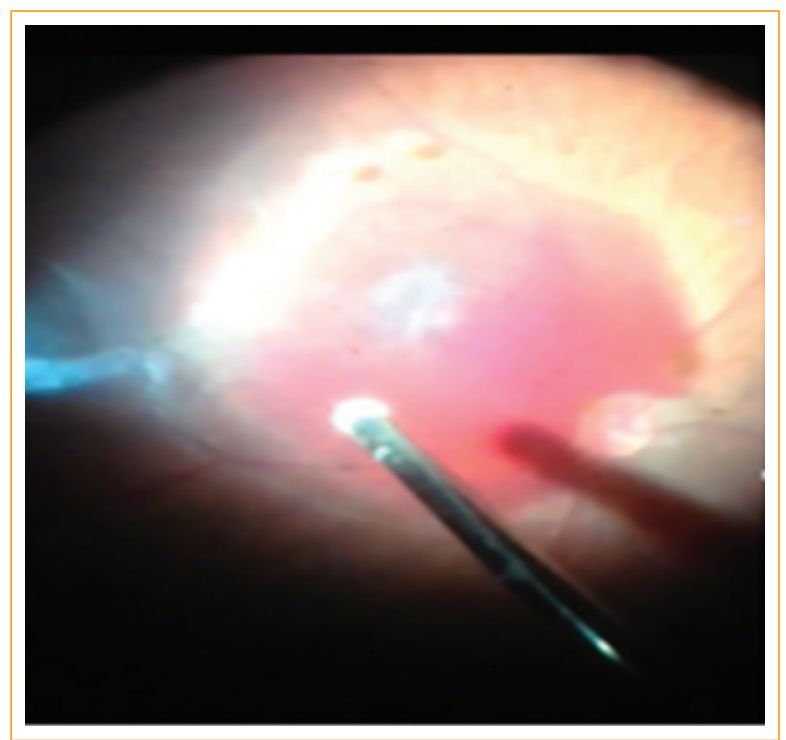

Figura 6. Polo posterior del ojo derecho durante la vitrectomía vía pars plana.

muy graves para aquellos declarados culpables en los tribunales de haber maltratado a niños. En los juzgados de lo Civil, el Estado podrá imponer por último adopciones confidenciales forzosas para cortar los lazos entre los hijos y sus padres a aquellos declarados culpables en cualquiera de los dos tipos de juzgados, generalmente enviando a los niños para ser custodiados por el Desarrollo Integral de la Familia ${ }^{24}$.

Aquel padre que ejerza habitualmente violencia física o psíquica sobre el menor que tiene sujeto a potestad se enfrentará a una pena de entre 6 meses y 3 años de prisión; además, si el juez lo estima adecuado, impondrá una inhabilitación especial de la patria potestad de $1 \mathrm{a}$
5 años, sin perjuicio de las penas que puedan corresponder por los delitos de violencia física o psíquicā.

Dejando a un lado los supuestos relacionados con la patria potestad, aquella persona que por cualquier medio o procedimiento cause a una persona menor de 12 años una lesión que menoscabe su integridad corporal o su salud física o mental, siempre que esta requiera objetivamente para su sanidad un tratamiento médico o quirúrgico, podrá ser castigada con una pena de prisión de 2 a 5 años ${ }^{25}$.

Los niños en México y Latinoamérica están desperotegidos de la violencia, pero no solo de la delincuencia, sino principalmente de la que ocurre dentro de sus casas. En México, solo Ciudad de México, Zacateccas y Chiapas prohíben específicamente el castigo corporal en sus leyes, mientras que Guanajuato y Chiapas lo prohíben en sus códigos penales. Por contra los códigos penales de 14 entidades contemplan excusas para lesionar a los descendientes: ya sea porque el agresor estaba invadido de una "emoción violenta", porque no lo hizo con dolo o porque ejerció su «dèrecho a corregirlo".

El artículo 321 bis del Código Penal Federal dicta-que no se procederá contra quien culposamente (por imprudencia) ocasione lesiones u homicidio en agravio dē un ascendiente (padres) o descendiente consanguíneô en

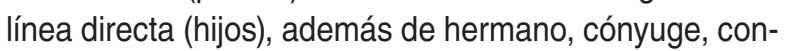
cubino, adoptante o adoptado, "salvo que el autop se encuentre bajo el efecto de bebidas embriagantes, de estupefacientes o psicotrópicos, sin que medie prescripción médica, o bien que no auxiliare a la víctima, 26

Párrafos casi idénticos figuran en los códigos de Baja California Sur, Chiapas, Morelos, Chihuahua, Nuevo León, Tlaxcala, Zacatecas, Michoacán, Guerrero y Ciudad de México.

El artículo 142 del Código Penal de Baja California Sur indica que se reducirá hasta la mitad el castigo a quien «en estado de emoción violenta cometa el delito de homicidio o de lesiones", y explica que existe emoción violenta cuando «en virtud de las circunstancias que desencadenaron el delito, se atenúa en forma considerable y trănsitoria" la capacidad del agresor para comprender loque hizo y actuar en consecuencia. Es el mismo casö de Guerrero, Tabasco, el Estado de México y Coahuila $\frac{27}{\mathrm{a}}$

Por su parte, Michoacán, Campeche y Ciudad de México reducen dos terceras partes de la pena a quienes cometan lesiones u homicidio «en estado de emoción violenta», definido por el Código Penal de Campeche como «una reacción motora, circulatoria y secretoria hacia un sentimiento de gran intensidad, el cual prođuce una perturbación psicológica transitoria que se manifiesta 
a través de formas violentas de expresión, falta de razonamiento, de discernimiento y de voluntad".

El Código Penal de la Ciudad de México, finalmente, define esta emoción como «cuando el sujeto activo del delito vive una intensa conmoción del ánimo que provoca un desorden del comportamiento, la pérdida del dominio de su capacidad reflexiva y la disminución de sus frenos inhibitorios» ${ }^{28}$.

Si bien dichas reglas aplican a cualquier familiar de los mencionados, los niños son más vulnerables a las lesiones y tienen menor capacidad de defenderse de sus parientes adultos, en particular cuando existe la creencia de que lastimar a un niño es «necesario» para educarlo. Esta creencia está institucionalizada en los Códigos Penales de los Estados de Hidalgo y Sonora, donde las lesiones forman parte del «derecho de corrección»y, por lo tanto, no causan penalidad.

«No son punibles los golpes inferidos en ejercicio del derecho de corregir, siempre y cuando no sean una forma habitual o reiterada de ejercer este derecho y no causen cualquiera de las lesiones previstas en el Artículo 140 de este Código", es decir, que causen daños a la salud, refiere el artículo 142 del Código de Hidalgo ${ }^{29}$. El de Sonora, por su parte, indica en su artículo 248 que las lesiones inferidas por quienes ejerzan la patria potestad o la tutela a los menores bajo su guarda «en ejercicio del derecho de corregir, no serán punibles si se tratare de las comprendidas en la fracción I del artículo 243», es decir, que no pongan en peligro la vida «y, además, el autor no abusare de ese derecho, corrigiendo con crueldad o con innecesaria frecuencia» ${ }^{30}$.

En nuestra serie de casos se tomó la decisión conjunta de realizar la denuncia; sin embargo, ninguna progresó, ya que las acciones de agresión fueron reiteradamente negadas por los presuntos responsables, de tal forma que no se logró un seguimiento legal.

\section{Conclusión}

Se requiere una amplia difusión del tema para fomentar la cultura de la denuncia y el seguimiento por parte del equipo multidisciplinario, las instituciones y los profesionales de la salud que intervienen en la atención de niños, para así evitar nuevas agresiones al menor. En nuestra serie, el diagnóstico clásico por interpretación de hallazgos del síndrome de maltrato infantil y el síndrome del niño sacudido fue altamente sugestivo; sin embargo, tiene tantas aristas e interpretaciones fisiopatológicas y legales que no pudo ser concluyente, y por tal motivo, emplear los términos «trauma craneal abusivo», «lesión cerebral adquirida» o «lesión por sacudida» pudiera ser una alternativa para la clasificación nosológica del maltrato infantil.

\section{Conflicto de intereses}

Los autores declaran no tener ningún conflict@ de intereses.

\section{Responsabilidades éticas}

Protección de personas y animales. Los autores declaran que para esta investigación no se han rêalizado experimentos en seres humanos ni en animales.

Confidencialidad de los datos. Los autores deçlaran que han seguido los protocolos de su centrô de trabajo sobre la publicación de datos de pacientes.

Derecho a la privacidad y consentimiento informado. Los autores han obtenido el consentimiênto informado de los pacientes y/o sujetos referidos en el artículo. Este documento obra en poder del autor de correspondencia.

\section{Bibliografía}

1. Levin AV. Retinal hemorrhage in abusive head trauma. Ped 2010;126(5):961-70.

2. Kempe $\mathrm{CH}$, Silverman $\mathrm{FN}$, Steele, BF, et al. The battered-child syndrome. JAMA. 1962;181(1):17-24.

3. Casado-Flores J, Díaz-Huertas JA, Martínez González C. Niños mâltratados. Madrid: Díaz de Santos; 1997. 388 p.

4. Cohen M, Lynne W, Bache B, Pragnell C. Carta abierta sobre el sindrome del bebé sacudido y los tribunales: una premisa falsa y defecturosa. Rev Electrónica Autops. 2015;13:13-6. Disponible en: http://rea.uninet. edu/index.php/ejautopsy/article/view/80

5. Rufo-Campos M. El síndrome del niño sacudido. Cuad Med Forense. 2006;12:39-45.

6. Simon JW, Zobal-Ratner J. A systematic review of the diagnostic accuracy of ocular signs in pediatric abusive head trauma. Evidence-Based Ophthalmol. 2011;12:26-7.

7. Fernández Couse G, Perea Corral J. Síndrome del maltrato infantíf Rev Cubana Pediatr. 2004;76(3). Disponible en: http://scielo.sld.cu/seielo. php?script=sci_arttext\&pid=S0034-75312004000300009\&lng=es\&num=iso\&tlng=en

8. Loredo-Abdalá A, Trejo-Hernández J, Melquiades-Parra I. Síndromène del niño sacudido. Forma extrema del abuso físico infantil. Acta Pediátrica México. 2014;32:177-9.

9. García Pérez J, Díaz Huertas J, Lirio Casero J. El síndrome dęniño zarandeado. Bienestar y Protección Infantil. 2003;2:13-23.

10. Hymel KP, Bandak PA, Partington MD, Winston KR. Abusive heä Gtrauma? A biomechanics-based approach. Child Maltreat. 1998;3:116-28.

11. Alexander R, Bennett T, Smith W. Incidence of impact trauma with eranial injuries ascribed to shaking. Am J Dis Child. 1990;144:724-6.

12. Talvik I, Alexander RC, Talvik T. Shaken baby syndrome and a שaby's cry. Acta Paediatr Int J Paediatr. 2008;97:782-5.

13. Koen WJ, Squier W. Shaken baby syndrome. Forensic Sci Reform Prot Innocent. 2017;6420:95-136.

14. Loredo-Abdalá A, Casas-Muñoz A, Trejo-Hernández J, Melquiades@ $९$ arra I, Martín-Martín V. Síndrome del niño sacudido: cuadro clínico y èyolución de 17 casos en el Instituto Nacional de Pediatría. Acta PediatiMex. 2015;36:72.

15. Starling SP, Holden JR, Jenny C. Abusive head trauma: the relationship of perpetrators to their victims. Pediatrics. 1995;95:259-62.

16. Organización Mundial de la Salud. Maltrato infantil. 2020. Disponible en: https://www.who.int/es/news-room/fact-sheets/detail/child-maltreatment 
17. Caffey J. On the theory and practica of shaking infants. Its potencial residual effects of permanent brain damage and mental retardation. Am J Dis Child. 1972;124:430-1.

18. Porras VR, Pérez V. Síndrome del niño sacudido: la importancia de las hemorragias retineanas y hematomas subdurales en sudiagnóstico. Acta Pediatr Costarric. 2002;16:2.

19. King WJ, MacKay M, Sirnick A. Shaken-baby syndrome in Canada; clinical characteristics and outcomes of hospital cases. CMAJ 2003;168:155-9.

20. Verdú F. En busca de la tríada. Gac. int. cienc. Forense. 2018. N.- 27 abril-junio. Disponible en: https://www.uv.es/gicf/1Ed2_Verdu_GICF_27.pd

21. Rufo M. El síndrome del niño sacudido. Cuad Med Forense. 2006:12:43-4

22. Corchado HS, Loredo-Abdalá A, Pérez Martínez A, Villa RA. El niño maltratado: obstáculos medicos y legales en el diagnóstico y asistencia integral. Bol Med Hosp Infant Mex. 2004;61:412-22.

23. Hymel K, Hall C. Diagnosing pediatric head trauma. Guidance for pediatricians on distinguishing between accidental and inflicted. Pediatrics Annals. 2005;34,358-70.

24. Loredo-Abdalá A, Trejo-Hernández J, Melquíades-Parra I. Síndrome de niño sacudido. Forma extrema del abuso físico infantil. Acta Pediatr Mex. 2011;32:177-9.

25. Código Penal para el Distrito Federal en Materia de Fuero Común, y para toda la República en Materia de Fuero Federal. (Consultado el 2 de agosto de 2020.) Disponible en: https://www.google.com/search?rlz $=1 \mathrm{C} 1 \mathrm{CH}$ BF_esMX892MX892\&biw=1366\&bih=625\&sxsrf=ALeKk01ZQwLo18Z1cl4g9rb0ymg44nuclw\%3A1596555745466\&ei=4YEpX9uJHliuswWg
26. Código Penal Federal de la Ciudad de México. Cámara de Diputados. H Congreso de la Unión. Título Decimonoveno. Capítulo III. Última reforma publicada DOF 24-01-2020. Disponible en: ПAttp:// www.diputados.gob.mx/LeyesBiblio/pdf_mov/Codigo_Penal_Federal.pdf

27. Código Penal de Baja California. H Congreso del Estado de BająCalifornia. Capítulo VI, Lesiones. Última reforma P.O. No. 49, Secc.III, 23 de octubre de 2015. Disponible en: http://www.ordenjuridico.gob.mx/苂ocumentos/Estatal/Baja\%20California/w080296.pdf

28. Codigo Penal de la Ciudad de Mexico, última reforma publicadáen la Gaceta Oficial de la Ciudad de México, el 31 de diciembre de 2018. Libro primero, Disposiciones generales, Título preliminar de los principios y garantías penales, Capítulo III, Reglas comunes para los delitos de homicidio y lesiones (reformado, g.o. 18 de marzo de 2011). Disponible en: https://docs.mexico.justia.com/estatales/hidalgo/codigo-penal-para-el-estado-de- hidalgo.pdf

29. Código Penal para el Estado de Hidalgo, última reforma publicada en el Periodico Oficial del 30 de julio de 2018. Capítulo II, Lesiones. Disponible en: http://www.congresohidalgo.gob.mx/biblioteca_leggislativa/Leyes/09Codigo\%20Penal\%20para\%20 el\%20Estado\%20de $\% 20$ Hidalgo.pdf

30. Código Penal del Estado de Sonora. Libro primero, Título decimosexto, Delitos contra la vida y la salud. Capítulo I, Lesiones. Disponible en: www.congresoson.gob.mx:81/Content/Doc_leyes/doc_443.pdf en: 